\section{BMJ Open} Ophthalmology

\title{
Role of corneal biomechanical properties in predicting of speed of myopic progression in children wearing orthokeratology lenses or single- vision spectacles
}

\author{
Kin Wan, ${ }^{1}$ Sin Wan Cheung, ${ }^{1}$ James S Wolffsohn, ${ }^{2}$ Janis B Orr, ${ }^{2}$ Pauline Cho ${ }^{1}$
}

\begin{abstract}
To cite: Wan K, Cheung SW, Wolffsohn JS, et al. Role of corneal biomechanical properties in predicting of speed of myopic progression in children wearing orthokeratology lenses or single-vision spectacles. BMJ Open Ophthalmology 2018;3:e000204. doi:10.1136/ bmjophth-2018-000204
\end{abstract}

- Additional material is published online only. To view please visit the journal online (http://dx.doi.org/10.1136/ bmjophth-2018-000204).

Received 10 August 2018 Revised 9 November 2018 Accepted 3 December 2018

A Check for updates

(C) Author(s) (or their employer(s)) 2018. Re-use permitted under CC BY-NC. No commercial re-use. See rights and permissions. Published by BMJ.

${ }^{1}$ School of Optometry, The Hong Kong Polytechnic University, Hong Kong, Hong Kong ${ }^{2}$ School of Life and Health Sciences, Aston University, Birmingham, UK

Correspondence to Mr Kin Wan; kkinwan@polyu. edu.hk

\section{ABSTRACT}

Objective To determine the characteristics of children who were likely to progress rapidly and gain the greatest benefit from orthokeratology (ortho-k) treatment.

Methods and analysis The files of 113 children who participated in two myopia control studies and wore either ortho-k lenses ( $\mathrm{n}=62$ ) or single-vision spectacles (SVS) $(n=51)$ were reviewed. Baseline cycloplegic subjective refraction, central corneal thickness, axial length, keratometry, intraocular pressure, corneal biomechanical properties and 24-month axial length data were retrieved and analysed.

Results Multivariate analysis showed that there was significant negative correlation between axial elongation and baseline age and corneal hysteresis $(p<0.05)$ in the SVS group. In the ortho-k group, only baseline age was significantly and negatively associated with axial elongation $(p<0.01)$.

Conclusion Corneal biomechanical properties and baseline age can predict the rate of axial elongation in myopic children. It may be beneficial for younger myopic children with low corneal hysteresis to commence ortho-k treatment as early as possible.

\section{INTRODUCTION}

Myopia is of particular concern due to its dramatic increase in prevalence worldwide. ${ }^{1}$ In Hong Kong, a study investigating myopia prevalence among 2883 primary schoolchildren between late 2005 and early 2010 reported that the prevalence of myopia was $18.3 \%$ and $61.5 \%$ at age 6 and 12, respectively, and that the prevalence of myopia higher than $-6.00 \mathrm{D}$ was $1.8 \% .^{2}$ The prevalence was much higher than the $9 \%$ reported at age $7-8$ a decade ago. ${ }^{3}$ Flitcroft suggested that the relatively low incidence of myopia at or before 6 years of age compared with that of older children was due to a secondary failure of the emmetropisation mechanisms. ${ }^{4}$

The association of ocular diseases with myopia is well recognised, with the OR of glaucoma increasing from 2.3 for low myopia

\section{Significance of the study}

What is already known about this subject?

- Corneal biomechanical properties are related to axial length.

- From the results of cross-sectional studies, lower corneal hysteresis is associated with longer axial length.

\section{What are the new findings?}

- In our retrospective study, a negative correlation between axial elongation and baseline corneal hysteresis $(\mathrm{CH})$ was revealed in single-vision spectacle (SVS) group.

- A significant difference in corneal biomechanical properties was observed between slow and fast progressing subgroups in the SVS group, suggesting lower $\mathrm{CH}$ and corneal resistant factor may be a risk factor for axial elongation.

\section{How might these results change the focus of} research or clinical practice?

Corneal biomechanical properties could be used as a screening parameter to identify fast progressors, who may gain the greatest benefit from orthokeratology treatment.

to 3.3 for moderate-to-high myopia $(\geq-3.00$ D).$^{5}$ A meta-analysis of seven cross-sectional studies and one case-control study showed that myopia was associated with both nuclear and posterior subcapsular cataracts. $^{6} \quad$ A study in Singapore reported strong association between myopia $>-6.00 \mathrm{D}$ and retinal changes, for example, staphyloma, chorioretinal atrophy and temporal peripapillary atrophy. $^{7}$

Since myopia can increase lifetime risk of ocular disease, which may lead to blindness, many studies have been conducted to investigate interventions to control myopia progression, one of which is orthokeratology (ortho-k). Ortho-k has been shown to be effective in slowing axial elongation (AE) by 
$36 \%-63 \%$ compared with subjects wearing single-vision spectacles (SVS) ${ }^{8-14}$ The variation in the level of myopia control reported by various studies may be attributable to differences in ages and refraction of the subjects recruited, resulting in differences in baseline characteristics. Mutti et $a l^{15}$ investigated the differences in characteristics between children who remained emmetropic and those who later developed myopia and suggested that longer axial length (AL), higher negative refractive error and higher relative hyperopic peripheral refractive error were associated with a faster rate of myopia progression.

Corneal biomechanical properties may also influence myopia progression. Roberts $\mathrm{et}^{\mathrm{al}} \mathrm{l}^{16}$ studied differences in corneal biomechanical properties between myopic and hyperopic eyes and reported there was a significant difference in corneal hysteresis $(\mathrm{CH})$, corneal resistance factor $(\mathrm{CRF})$ and several parameters derived from the waveform signal. $\mathrm{CH}$ and $\mathrm{CRF}$ are specific outputs from the Ocular Response Analyzer (ORA; Reichert Ophthalmic Instruments, Buffalo, New York, USA). CH refers to the ability of the corneal tissue to dissipate energy, and CRF refers to an indicator of the overall resistance of the cornea. ${ }^{1718} \mathrm{~A}$ higher $\mathrm{CH}$ and $\mathrm{CRF}$ implies a better ability to resist from deformation by pressure. Although associations between $\mathrm{AL}$ and corneal biomechanical properties have been reported in children and adults, ${ }^{19-23}$ to our knowledge, there are no published studies comparing the effect of ocular biomechanical properties on $\mathrm{AE}$ in myopic children wearing ortho-k lenses and SVS. This study aimed to address this gap and investigate the differences and association with $\mathrm{AE}$ in baseline age and ocular characteristics. The ocular characteristics of subjects demonstrating fast and slow progression were also compared for both the ortho-k and SVS wearers.

\section{METHODS}

All the data were retrieved from two previous studies, ROMIO $^{8}$ and TO-SEE. ${ }^{9}$ All control subjects were corrected by spherical SVS made of CR39 material with refractive index of 1.56 (Hong Kong Optical Lens, Hong Kong, China). All ortho-k subjects were fitted with spherical $(\mathrm{ROMIO})^{8}$ or toric (TO-SEE) ${ }^{9}$ Menicon Z Night lenses (NKL Contactlenzen, Emmen, The Netherlands). Lens prescription was aided by a computer program provided by the lens manufacturers in both studies. Full refractive correction was provided for all subjects and the habitual prescription was updated if the monocular visual acuity was worse than 0.18 (logarithm of the minimum angle of resolution $(\log$ MAR) $)($ Snellen $6 / 9)$ or residual myopia/astigmatism exceeded $0.50 \mathrm{D}$ at any visit after stabilisation of treatment. Measurements were repeated every 6 months for 2 years after the baseline visit. Table 1 shows a summary of the inclusion and exclusion criteria of ROMIO and TO-SEE studies. ${ }^{89}$

Baseline data (cycloplegic subjective refraction, central corneal thickness (CCT), AL, keratometry, intraocular

\begin{tabular}{|c|c|}
\hline Inclusion criteria & Exclusion criteria \\
\hline $\begin{array}{l}\text { ROMIO } \\
6-10 \text { years (inclusive) } \\
\text { Myopia: between } 0.50 \mathrm{D} \text { and } 4.00 \mathrm{D} \text { in at least one eye } \\
\text { Astigmatism: }<1.50 \mathrm{D} \text {; with-the-rule astigmatism (axes } \\
180 \pm 30 \text { ) } \leq 1.25 \mathrm{D} \text {; astigmatism of other axes } \leq 0.50 \mathrm{D} \text { in both } \\
\text { eyes } \\
\text { Spherical equivalent }(\mathrm{SE}):>0.50 \mathrm{D} \text { and } \leq 4.50 \mathrm{D} \text { in both eyes } \\
\text { Anisometropia: } \leq 1.50 \mathrm{D} \\
\text { Best-corrected logMAR visual acuity } 0.10 \text { or better in both } \\
\text { eyes } \\
\text { Symmetrical corneal topography with corneal toricity }<2.00 \\
\mathrm{D} \text { in either eye }\end{array}$ & $\begin{array}{l}\text { ROMIO and TO-SEE } \\
\text { Strabismus at distance or near } \\
\text { Previous experience in contact lens wear or myopia control } \\
\text { treatment (eg, refractive therapy or progressive spectacles) } \\
\text { Contraindication for contact lens wear and orthokeratology } \\
\text { (eg, limbus to limbus corneal cylinder and dislocated } \\
\text { corneal apex) } \\
\text { Previous history of ocular surgery, trauma or chronic ocular } \\
\text { disease } \\
\text { Concurrent use of medications that may affect tear quality } \\
\text { Systemic or ocular conditions that may affect tear } \\
\text { quality or contact lens wear (eg, allergy and concurrent } \\
\text { medication) or that may affect refractive development (eg, } \\
\text { Down syndrome, ptosis) } \\
\text { Poor compliance to tests (eg, poor fixation in noncontact } \\
\text { tonometry or intolerance of lens wear) } \\
\text { Not willing to comply with the allocated treatment and } \\
\text { follow-up schedule }\end{array}$ \\
\hline $\begin{array}{l}\text { TO-SEE } \\
\text { 6-12 years (inclusive) } \\
\text { Myopia: between } 0.50 \mathrm{D} \text { to } 5.00 \mathrm{D} \\
\text { Astigmatism (with-the-rule astigmatism): } 1.25-3.50 \mathrm{D} \text {; (axes } \\
180 \pm 20 \text { ) } \\
\text { Anisometropia: } \leq 1.50 \mathrm{D} \\
\text { Best-corrected logMAR visual acuity } 0.10 \text { or better in both } \\
\text { eyes }\end{array}$ & \\
\hline
\end{tabular}

$\log M A R$, logarithm of the minimum angle of resolution. 
pressure (IOP) and corneal biomechanical properties) and 24-month AL data were retrieved and analysed. The refractive examination was conducted according to standard clinical protocol which resulted in the maximum plus lens without detriment to the visual acuity steps. Binocular balancing with prism dissociation was also performed to ensure maximum visual acuity could be achieved. CCT and keratometry were measured using the Pentacam (V.1.12, Oculus, Arlington, Washington, USA). AL was measured with partial coherence laser interferometry (IOL Master; Carl Zeiss, Oberkochen, Germany). Goldmann-correlated intraocular pressure (IOPg), corneal compensated intraocular pressure (IOPcc) and corneal biomechanical properties were measured with the ORA (V.2.00, Reichert Ophthalmic Instruments). Valid ORA data were defined as those with a waveform score $\geq 4.0$. The average of four valid ORA data readings was used in data analyses. To further analyse the association between $\mathrm{AE}$ and ocular characteristics, ocular characteristics of two subgroups (10 subjects with the fastest (control: $0.91 \leq \mathrm{AE} \leq 1.15 \mathrm{~mm}$; Ortho-k: $0.58 \leq \mathrm{AE} \leq$ $0.87 \mathrm{~mm}$ ) and 10 subjects with the slowest (control: $0.18 \leq \mathrm{AE} \leq 0.43 \mathrm{~mm}$; Ortho-k: $-0.26 \leq \mathrm{AE} \leq 0.07 \mathrm{~mm}$ ) $\mathrm{AE}$ in each group of subjects) were compared.

\section{Treatment of data}

SPSS V.22.0 (IBM) was used to perform statistical analysis. The data from $\mathrm{ROMIO}^{8}$ and TO-SEE ${ }^{9}$ were combined since the protocol of data collection, study period and most inclusion criteria, except for high astigmatism power for TO-SEE study, was the same. The data sets followed a normal distribution (Kolmogorov-Smirnov tests, $p>0.05$ ), hence parametric tests were used for analyses. Fisher's exact probability test was performed to test the sex difference between ortho-k and SVS groups. Other differences in the baseline characteristics between the ortho-k and SVS subjects were tested using unpaired t-tests. Since AE was significantly slower ${ }^{8} 9$ in subjects undergoing ortho-k than those wearing SVS, ortho-k and SVS groups were analysed separately. Stepwise multiple linear regression was used to analyse the effect of AE over 24 months in ortho-k and SVS groups. To identify potential differences in ocular characteristics between fast and slow progressors, subgroup analysis was also performed: one-way analysis of variance (ANOVA) (or one-way analysis of covariance (ANCOVA), where indicated) was used to compare the ocular characteristics of 10 subjects with the fastest and 10 subjects with the slowest $\mathrm{AE}$ over 2 years in the two groups of subjects. Except for refractive cylinder (Shapiro-Wilk tests, $\mathrm{p}<0.001$ ), all the data from the subgroups followed normal distribution (Shapiro-Wilk tests, $\mathrm{p}>0.05$ ). Post-hoc tests with Bonferroni correction were performed if significant differences were found.

\section{RESULTS}

In total, 113 (ortho-k: 62, SVS: 51) of 136 (ortho-k: 72, SVS: 64) subjects had valid ORA data. Table 2 presents the initial age and ocular characteristics of these 113 subjects.

There were no significant differences in the demographic data between the two groups of subjects $(p>0.05)$, or between males/females (unpaired t-test, $\mathrm{p}>0.05$ ). AE over 24 months was significantly associated with initial age (adjusted $\mathrm{R}^{2}=0.085, \quad \mathrm{~F}_{1,60}=6.672$, standardised beta $=-0.316, p=0.012$ ) but not with initial AL, corneal curvature, CCT, IOPg, IOPcc, CRF or CH of the subjects

Table 2 Baseline age and ocular characteristics of subjects

\begin{tabular}{llllll} 
& Ortho-k $(\mathbf{n}=\mathbf{6 2})$ & SD & $\begin{array}{l}\text { SVS } \\
(\mathbf{n = 5 1 )}\end{array}$ & SD & P value* $^{*}$ \\
\hline Age (years) & 9.06 & 1.17 & 8.69 & 1.26 & 0.411 \\
\hline Gender (F/M) & $29 / 33$ & - & $23 / 28$ & - & $0.999 \dagger$ \\
\hline Sub. Sph (D) & -2.25 & 1.05 & -2.09 & 0.89 & 0.293 \\
\hline Sub. Cyl. (D) & -0.98 & 0.92 & -0.97 & 1.00 & 0.303 \\
\hline SER (D) & -2.74 & 1.24 & -2.57 & 0.96 & 0.101 \\
\hline AL $(\mathrm{mm})$ & 24.44 & 0.86 & 24.29 & 0.84 & 0.833 \\
\hline Mean K (D) & 43.62 & 1.92 & 44.10 & 1.51 & 0.746 \\
\hline CCT $(\mu \mathrm{m})$ & 569 & 27.45 & 577 & 31.00 & 0.222 \\
\hline IOPg $(\mathrm{mm} \mathrm{Hg})$ & 14.88 & 2.32 & 15.10 & 2.35 & 0.890 \\
\hline IOPcc $(\mathrm{mm} \mathrm{Hg})$ & 14.99 & 2.02 & 14.66 & 2.22 & 0.455 \\
\hline CRF $(\mathrm{mm} \mathrm{Hg})$ & 10.59 & 1.45 & 11.06 & 1.41 & 0.703 \\
CH $(\mathrm{mm} \mathrm{Hg})$ & 10.82 & 1.25 & 11.31 & 1.30 & 0.959 \\
\hline
\end{tabular}

*Probability values of $t$-test for difference between ortho-k and SVS groups.

†Probability values of Fisher's exact probability test for difference between ortho-k and SVS groups.

Sub. Sph, subjective sphere; Sub. Cyl, subjcetive cylinder; SER, spherical equivalent refraction; AL, axial length; CCT, central corneal thickness; $\mathrm{CH}$, corneal hysteresis; CRF, corneal resistant factor; IOPg, intraocular pressure; IOPcc, corneal compensated intraocular pressure; Ortho-K, orthokeratology group; SVS single-vision spectacles group. 


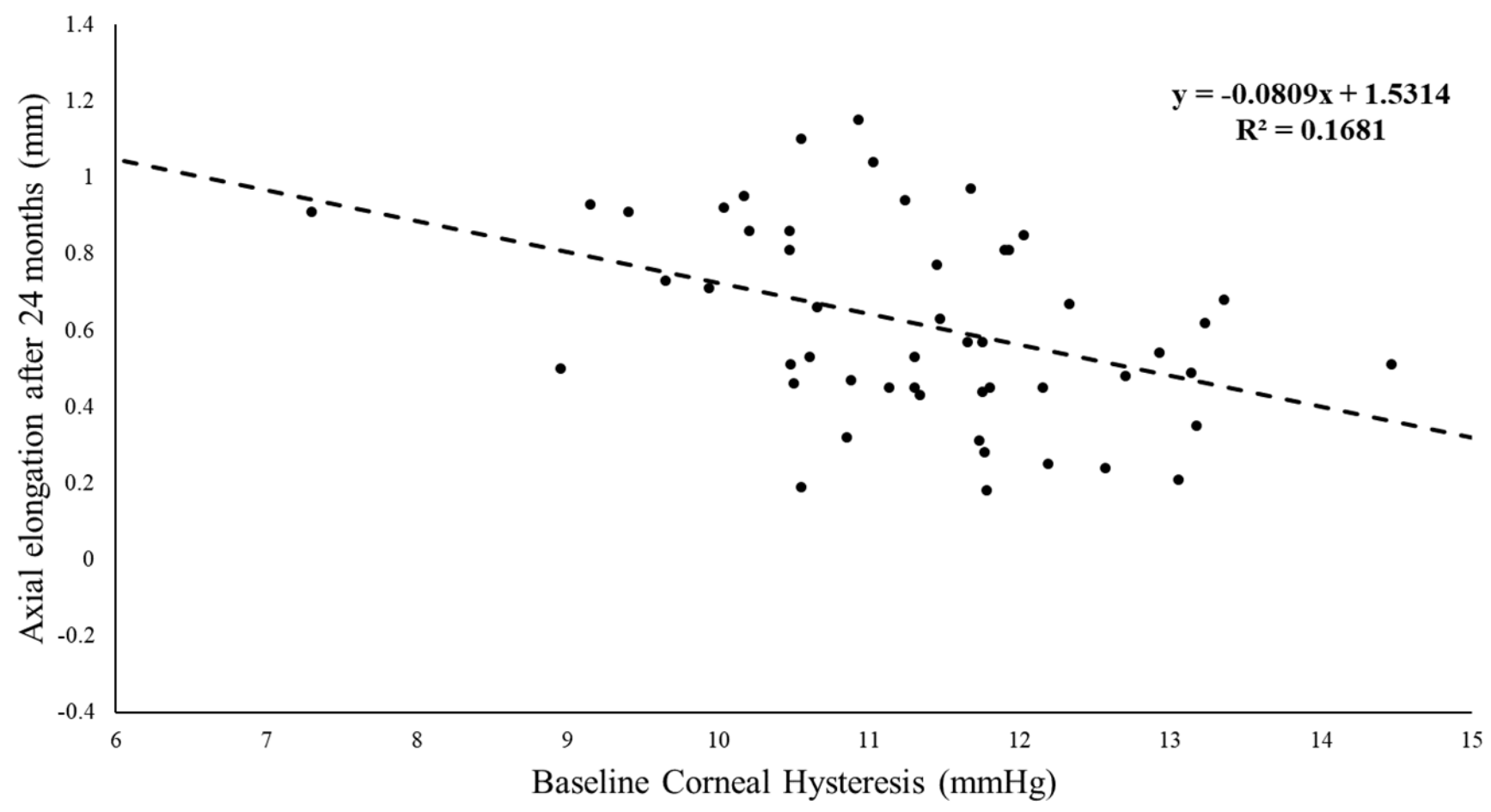

Figure 1 Association between corneal hysteresis and axial elongation in single-vision spectacle group $(n=51)$.

in the ortho-k group (partial r: -0.240 to $0.091, \mathrm{p}>0.05$ ). However, in the SVS group, AE was significantly associated with initial age (standardised beta $=-0.327, p=0.015$ ) and $\mathrm{CH}$ (standardised beta $=-0.316, \mathrm{p}=0.018$ ) (adjusted $\left.\mathrm{R}^{2}=0.236, \mathrm{~F}_{2,48}=8.741, \mathrm{p}=0.001\right)$, but not with initial AL, corneal curvature, CCT, IOPg, IOPcc or CRF (partial r:
-0.191 to $0.084, p>0.05)$. Figure 1 and 2 present the plot of the association between $\mathrm{CH}$ and $\mathrm{AE}$ after 24 months in SVS and ortho-k group, respectively.

Baseline data of the 40 subjects with the fastest and slowest $\mathrm{AE}$ in the two study groups are presented in table 3 .

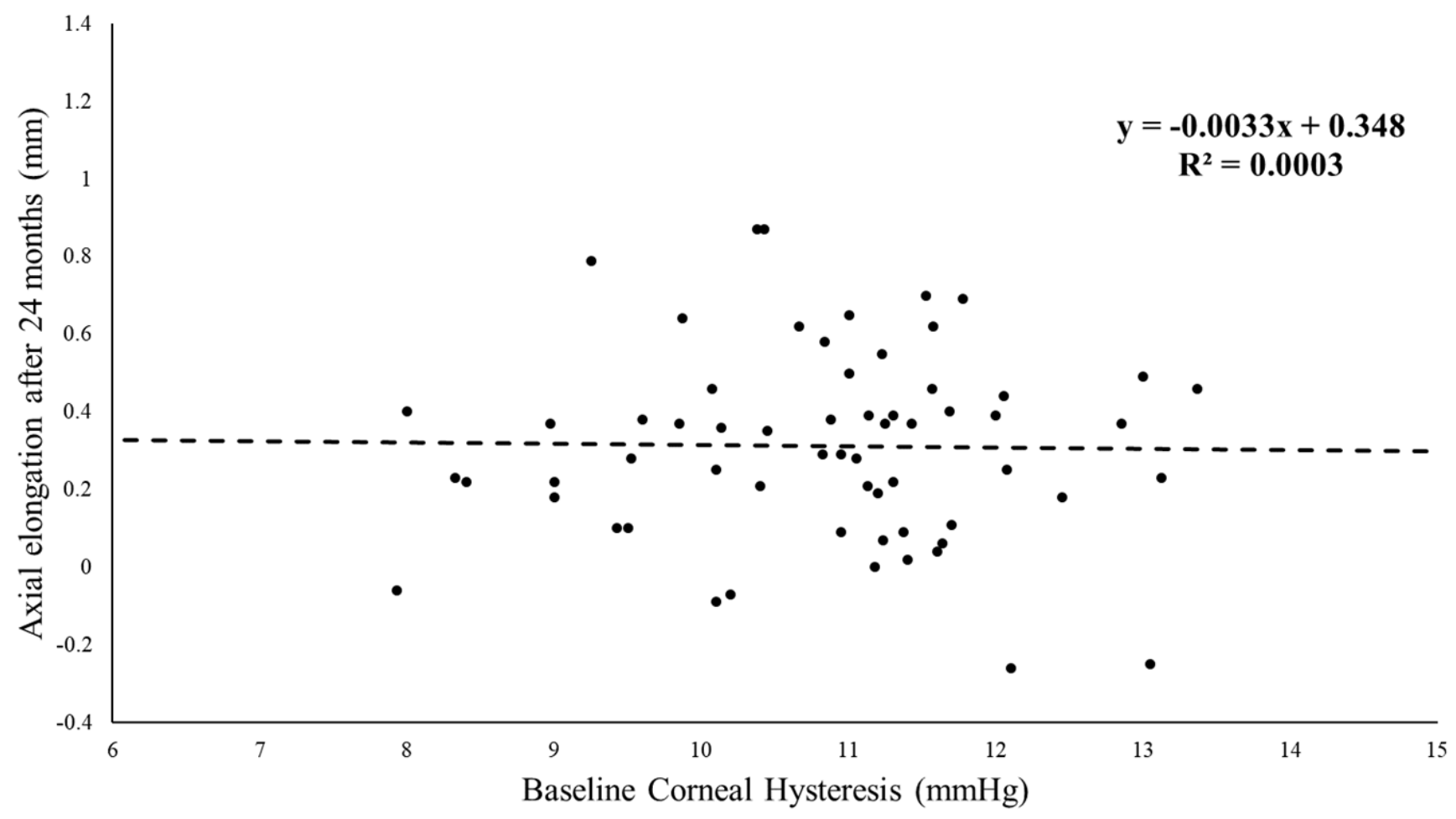

Figure 2 Association between corneal hysteresis and axial elongation in ortho-k group ( $n=62)$. 
Table 3 Baseline age and ocular characteristics of 10 subjects with the fastest and 10 subjects with the slowest axial elongation (AE) after 24 months of monitoring

\begin{tabular}{|c|c|c|c|c|c|c|c|c|c|}
\hline & \multicolumn{4}{|c|}{ Ortho-k } & \multicolumn{4}{|l|}{ svs } & \multirow[b]{3}{*}{$P$ value } \\
\hline & Slow & SD & Fast & SD & Slow & SD & Fast & SD & \\
\hline & \multicolumn{2}{|l|}{$\mathrm{N}=10$} & \multicolumn{2}{|l|}{$N=10$} & $\mathrm{~N}=10$ & & \multicolumn{2}{|l|}{$N=10$} & \\
\hline$A E(\mathrm{~mm})$ & -0.05 & 0.12 & 0.70 & 0.10 & 0.28 & 0.08 & 0.98 & 0.09 & $0.000 \dagger$ \\
\hline Age (years) & 9.7 & 1.16 & 8.2 & 0.63 & 9.4 & 1.07 & 7.7 & 1.16 & $0.000 \dagger$ \\
\hline Gender (F/M) & $7 / 3$ & & $5 / 5$ & & $7 / 3$ & & $4 / 6$ & & 0.528 \\
\hline Sub. Sph (D) & -2.70 & 1.33 & -1.75 & 1.02 & -2.03 & 1.17 & -1.85 & 0.92 & 0.242 \\
\hline Sub. Cyl. (D) & -1.48 & 1.20 & -0.73 & 0.78 & -1.03 & 0.84 & -1.05 & 1.11 & 0.423 \\
\hline SER (D) & -3.44 & 1.67 & -2.11 & 0.99 & -2.54 & 1.21 & -2.38 & 1.02 & 0.116 \\
\hline $\mathrm{AL}(\mathrm{mm})$ & 24.44 & 0.96 & 24.13 & 1.04 & 24.05 & 1.07 & 24.09 & 0.95 & 0.811 \\
\hline Mean K (D) & 44.36 & 1.37 & 43.69 & 1.50 & 44.25 & 1.95 & 44.11 & 1.49 & 0.794 \\
\hline $\mathrm{CCT}(\mu \mathrm{m})$ & 566 & 37.83 & 580 & 21.15 & 583 & 34.15 & 565 & 24.41 & 0.430 \\
\hline $\mathrm{IOPg}(\mathrm{mm} \mathrm{Hg})$ & 15.82 & 2.92 & 14.76 & 2.22 & 15.53 & 1.87 & 14.12 & 2.27 & 0.373 \\
\hline IOPcc $(\mathrm{mm} \mathrm{Hg})$ & 15.57 & 2.65 & 14.99 & 1.99 & 14.39 & 2.05 & 15.07 & 2.54 & 0.735 \\
\hline $\mathrm{CRF}(\mathrm{mm} \mathrm{Hg})$ & 11.06 & 1.59 & 10.48 & 1.00 & 11.69 & 0.80 & 9.78 & 1.20 & $0.007 \ddagger$ \\
\hline $\mathrm{CH}(\mathrm{mm} \mathrm{Hg})$ & 11.04 & 1.38 & 10.73 & 0.79 & 11.90 & 0.87 & 10.15 & 1.28 & $0.011 \ddagger$ \\
\hline
\end{tabular}

*Probability values of analysis of variancefor difference between ortho-k and SVS groups.

†Significant difference between fast and slow progressors in both ortho-k and SVS groups.

$\mathrm{AL}$, axial length; CCT, central corneal thickness; $\mathrm{CH}$, corneal hysteresis; CRF, corneal resistant factor; IOPg, intraocular pressure; IOPcc,

corneal compensated intraocular pressure; Ortho-K, orthokeratology group; SVS single-vision spectacles group.

AE differed among the four subgroups (one-way ANOVA, $\mathrm{p}<0.001)$. The AE was significantly lower in the ortho-k subjects compared with the SVS subjects in both subgroups $(\mathrm{p}<0.001)$.

There were significant differences in baseline age among the four subgroups (one-way ANOVA, $\mathrm{p}<0.05$ ). Post-hoc tests showed that the rapid progressing subgroups were about 1.5-1.7 years younger than those in the slow progressing subgroups for both ortho-k and SVS groups $(p<0.05)$. There was no significant difference in age between the ortho-k and SVS subjects in the fast progressing $(\mathrm{p}>0.99)$ and slow progressing subgroups ( $p>0.99)$. After controlling for age, there were significant differences in CH and CRF among the four subgroups (one-way ANCOVAs, $\mathrm{p}<0.05$ ). Post-hoc tests revealed that a significant difference in $\mathrm{CH}$ and $\mathrm{CRF}$ existed between the fast and slow progressing subgroups in the SVS subjects $(\mathrm{p}<0.05)$, but not between the ortho-k subjects $(\mathrm{p}>0.99)$.

\section{DISCUSSION}

The results of the current study showed that initial age was significantly associated with the AL elongation in both ortho-k and SVS groups. Subjects who were younger tended to progress faster than those who were older. This association has been previously reported ${ }^{24}$ and was in agreement with other reports on myopic children. ${ }^{25-27}$ Two previous studies conducted in Singapore reported that children with younger baseline age had faster myopia progression. ${ }^{26} 27$ Another study with ethnically diverse subjects also reported a similar result. ${ }^{25}$
In addition to initial age, $\mathrm{CH}$ was also associated with $\mathrm{AE}$ in the SVS group, but not in the ortho-k group. This association between AL and corneal biomechanical properties has been reported in several previous cross-sectional studies. ${ }^{192-23}{ }^{28}$ Chang et $a l^{22}$ reported that longer $\mathrm{AL}$ was associated with lower $\mathrm{CH}$ and $\mathrm{CRF}$ values in myopic children and there was a significant correlation between the difference of $\mathrm{AL}$ and $\mathrm{CH}$ between the two eyes of each subject. This might suggest that differences in corneal biomechanical properties may indicate generalised structural differences between eyes. Song et $a l^{21}$ also demonstrated an association between $\mathrm{AL}$ and corneal biomechanical properties in secondary school children. Shen $e t a l^{28}$ investigated the corneal biomechanical properties of adults with high myopia and showed significantly lower CH. Both Del Buey et $a l^{19}$ and Jiang $e t$ $a l^{33}$ also reported that $\mathrm{CH}$ in high myopes was decreased compared with low and non-myopic subjects. However, these were cross-sectional studies and, although they showed that longer AL or higher myopia was associated with lower $\mathrm{CH}$, they were not able to determine if low $\mathrm{CH}$ is a potential risk factor for AE. The current study suggests that in addition to initial age low $\mathrm{CH}$ may be a risk factor for $\mathrm{AE}$ in young myopic children not undergoing myopia control treatment.

Hysteresis is a physical property referred to the ability to dampen pressure changes. A lower $\mathrm{CH}$ value may imply a poor ability to resist the stretch in response to distending IOP stress. ${ }^{29}$ The corneal hysteresis might reflect the constitution of its extracellular matrix (ECM), which could be hypothesised to be related to the ECM 
composition of posterior ocular tissues. ${ }^{30}$ IOP had shown to be one mechanical factor that could lead to AE. ${ }^{31} 32$ Elevations in IOP had shown to be associated with $\mathrm{AE}$ of the eye. ${ }^{33-36}$ Many daily life activities including eye rubbing, ${ }^{37}$ forced eyelid closure ${ }^{38}$ and isometric exercises, ${ }^{39}$ can increase IOP within a short period of time. The relatively poor ability to resist the ocular deformation by sudden increase in IOP for subjects with low $\mathrm{CH}$ could be the reason to explain the negative association of $\mathrm{CH}$ and AE. In the ortho-k group, only initial age was associated with $\mathrm{AE}$. This result is in agreement with previous studies, which reported that younger ortho-k wearers tended to progress faster. ${ }^{40}{ }^{41}$ The mechanism leading to slowing down of AE by use of ortho-k is still unclear. It has been suggested that ortho-k increases myopic peripheral defocus ${ }^{42}$ which may lead to retardation of myopia progression. The amount of induced peripheral defocus varies individually, and it may not be associated with $\mathrm{CH}$ of individual subjects. This may help to explain why the effect of baseline $\mathrm{CH}$ was masked in the ortho-k group.

To further investigate the relationship between AL and ocular characteristics, baseline characteristics between 10 subjects with the fastest (fast progressor) and 10 subjects with the slowest (slow progressor) AE over 2 years in the two treatment groups were also compared. In addition to initial age, a significant difference in corneal biomechanical properties between fast and slow progressors in the SVS group was observed. CH and CRF were significantly higher in the slow progressors. The reproducibility and repeatability of the ORA were documented by published literatures in adults and children. ${ }^{43}{ }^{44}$ The reported intra-examiner reliability ranged from 0.78 to 0.93 (good to excellent) ${ }^{43}$ The reported mean \pm SD test-retest differences of children in $\mathrm{CH}$ and $\mathrm{CRF}$ were $0.36 \pm 1.14 \mathrm{~mm} \mathrm{Hg}$ and $0.18 \pm 1.04 \mathrm{~mm} \mathrm{Hg}$, respectively, in spectacle group and $-0.06 \pm 0.75$ and $0.06 \pm 0.77 \mathrm{~mm} \mathrm{Hg}$, respectively, in ortho-k group. ${ }^{44}$ These results showed that the $84 \%$ $($ mean $+1 \mathrm{SD})$ of subjects would expect to exhibit retest changes of corneal biomechanical properties of $<1.5 \mathrm{~mm}$ $\mathrm{Hg}$ in spectacle group and $0.83 \mathrm{~mm} \mathrm{Hg}$ in ortho-k group. The mean differences of $\mathrm{CH}$ and $\mathrm{CRF}$, between fast and slow progressors, in SVS group, which yielded statistically significance, were 1.75 and $1.91 \mathrm{~mm} \mathrm{Hg}$, respectively. In other words, on the basis of probability, such differences would more than likely represent a genuine difference rather than measurement noise.

Their initial AL, corneal curvature and CCT, which were previously reported as factors likely to affect $\mathrm{CH}$ and CRF measurements, ${ }^{45}{ }^{46}$ were matched in this study. Age was also reported to be a factor affecting $\mathrm{CH}$, and a significant difference of baseline age between the subgroups was also observed in the current study. Kida $e t$ $a l^{47}$ concluded there was an inverse relationship between age and $\mathrm{CH}$ in adults, and noted a similar association for CRF. However, Kirwan $e t a l^{48}$ did not find any significant correlation between age and $\mathrm{CH}$. In the current study, even after controlling for initial age, CH and CRF remained significantly lower in the fast progressors in the
SVS group, but this difference was not observed in the ortho-k group. The mean AE of the fast progressors in the SVS group was $0.98 \mathrm{~mm}$ after 24 months. If the rate of progression remained the same, it could be predicted that these subjects would most likely suffer from moderate to high myopia in later life. In the previous reports, $\mathrm{CH}$ was lower in moderate to high myopia subjects. ${ }^{192328}$ This result was indirectly in agreement with previous reports and provided further evidence that low $\mathrm{CH}$ may be a risk factor of myopia progression. The stepwise multiple linear regression model revealed that $\mathrm{CH}$ was associated with $\mathrm{AE}$ after 24 months in SVS group, but not in the ortho-k group.

From the result, patients, especially those with low $\mathrm{CH}$, should benefit from commencing ortho-k treatment as early as possible, to minimise the risk of myopia progression. Further longitudinal work, with larger sample sizes, would be required to confirm the role of $\mathrm{CH}$ and $\mathrm{CRF}$ in myopia progression and to determine the cut-off of $\mathrm{CH}$ and CRF points in order to determine the risk of myopia progression.

\section{CONCLUSION}

Subjects having slower myopia progression were significantly older than those with fast progression, irrespective of their form of refractive correction (SVS or ortho-k). In addition, significant differences in $\mathrm{CH}$ and $\mathrm{CRF}$ were observed between slow and fast progressing subgroups in the SVS group, suggesting lower CH and CRF may be a risk factor for AE.

Acknowledgements The authors thank Dr Maureen Boost for her advice in the preparation of the manuscript.

Contributors CP planned the current study. WK and CSW analysed the data and interpreted the results. WK wrote the first draft of the paper. CP, CSW, WJS and OJB reviewed the manuscript.

Funding This study was supported by the Research Residency Scheme from The School of Optometry, The Hong Kong Polytechnic University and a Collaborative Research Agreement (ZG3Z) between The Hong Kong Polytechnic University and Menicon Co Ltd., Japan, which supports a PhD student (W.K). The two previous studies were supported by a Collaborative Research Agreements between PolyU and Menicon Co Ltd., Japan (ZG13 and ZG30: ROMIO and TO-SEE studies).

\section{Competing interests None declared.}

Patient consent for publication Parental/guardian consent obtained.

Ethics approval Both studies were approved by the Departmental Research Committee of the School of Optometry of The Hong Kong Polytechnic University and conducted in accordance with the tenets of the Declaration of Helsinki.

Provenance and peer review Not commissioned; externally peer reviewed.

Open access This is an open access article distributed in accordance with the Creative Commons Attribution Non Commercial (CC BY-NC 4.0) license, which permits others to distribute, remix, adapt, build upon this work non-commercially, and license their derivative works on different terms, provided the original work is properly cited, appropriate credit is given, any changes made indicated, and the use is non-commercial. See: http://creativecommons.org/licenses/by-nc/4.0

\section{REFERENCES}

1. Holden B, Sankaridurg P, Smith E, et al. Myopia, an underrated global challenge to vision: where the current data takes us on myopia control. Eye 2014;28:142-6. 
2. Lam CS, Lam CH, Cheng SC, et al. Prevalence of myopia among Hong Kong Chinese schoolchildren: changes over two decades. Ophthalmic Physiol Opt 2012;32:17-24.

3. Edwards $\mathrm{MH}$. The development of myopia in Hong Kong children between the ages of 7 and 12 years: a five-year longitudinal study. Ophthalmic Physiol Opt 1999;19:286-94.

4. Flitcroft DI. Emmetropisation and the aetiology of refractive errors. Eye 2014;28:169-79.

5. Mitchell P, Hourihan F, Sandbach J. The relationship between glaucoma and myopia: the blue mountains eye study. Ophthalmol 1999;106:2010-5.

6. Pan CW, Cheng CY, Saw SM, et al. Myopia and age-related cataract: a systematic review and meta-analysis. Am J Ophthalmo 2013;156:1021-33.

7. Chang L, Pan CW, Ohno-Matsui K, et al. Myopia-related fundus changes in Singapore adults with high myopia. Am J Ophthalmol 2013;155:991-9.

8. Cho P, Cheung S-W. Retardation of myopia in orthokeratology (ROMIO) study: a 2-year randomized clinical trial. Invest Ophthalmol Vis Sci 2012;53:7077-85.

9. Chen C, Cheung SW, Cho P. Myopia control using toric orthokeratology (TO-SEE study). Invest Ophthalmol Vis Sci 2013;54:6510-7.

10. Walline JJ, Jones LA, Sinnott LT. Corneal reshaping and myopia progression. Br J Ophthalmol 2009;93:1181-5.

11. Kakita T, Hiraoka T, Oshika T. Influence of overnight orthokeratology on axial elongation in childhood myopia. Invest Ophthalmol Vis Sci 2011;52:2170-4

12. Santodomingo-Rubido J, Villa-Collar C, Gilmartin B, et al. Myopia control with orthokeratology contact lenses in Spain: refractive and biometric changes. Invest Ophthalmol Vis Sci 2012;53:5060-5.

13. Charm J, Cho P. High myopia-partial reduction orthokeratology (HMPRO): study design. Cont Lens Anterior Eye 2013;36:164-70.

14. Cho P, Cheung SW, Edwards $M$. The longitudinal orthokeratology research in children (LORIC) in Hong Kong: a pilot study on refractive changes and myopic control. Curr Eye Res 2005;30:71-80.

15. Mutti DO, Hayes JR, Mitchell GL, et al. Refractive error, axial length, and relative peripheral refractive error before and after the onset of myopia. Invest Ophthalmol Vis Sci 2007;48:2510-9.

16. Roberts CJ, Reinstein DZ, Archer TJ, et al. Comparison of ocular biomechanical response parameters in myopic and hyperopic eyes using dynamic bidirectional applanation analysis. J Cataract Refract Surg 2014;40:929-36.

17. Luce DA. Determining in vivo biomechanical properties of the cornea with an ocular response analyzer. J Cataract Refract Surg 2005;31:156-62.

18. Shah S, Laiquzzaman M, Cunliffe I, et al. The use of the Reichert ocular response analyser to establish the relationship between ocular hysteresis, corneal resistance factor and central cornea thickness in normal eyes. Cont Lens Anterior Eye 2006;29:257-62.

19. del Buey MA, Lavilla L, Ascaso FJ, et al. Assessment of corneal biomechanical properties and intraocular pressure in myopic Spanish healthy population. J Ophthalmol 2014;2014:1-6.

20. Shen M, Fan F, Xue A, et al. Biomechanical Properties of the cornea in high myopia. Vision Res 2008;48:2167-71.

21. Song Y, Congdon N, Li L, et al. Corneal hysteresis and axial length among Chinese secondary school children: the Xichang Pediatric Refractive Error Study (X-PRES) report no. 4. Am J Ophthalmol 2008;145:819-26.

22. Chang PY, Chang SW, Wang JY. Assessment of corneal biomechanical properties and intraocular pressure with the ocular response analyzer in childhood myopia. $\mathrm{Br} J$ Ophthalmol 2010;94:877-81.

23. Jiang Z, Shen M, Mao G, et al. Association between corneal biomechanical properties and myopia in Chinese subjects. Eye 2011;25:1083-9.

24. Cho P, Cheung S-W. Protective role of orthokeratology in reducing risk of rapid axial elongation: a reanalysis of data from the ROMIO and TO-SEE studies. Invest Ophthalmol Vis Sci 2017:58:1411-6.
25. Hyman L, Gwiazda J, Hussein M, et al. Relationship of age, sex, and ethnicity with myopia progression and axial elongation in the correction of myopia evaluation trial. Arch Ophthalmol 2005; 123:977-87.

26. Saw SM, Chua WH, Gazzard G, et al. Eye growth changes in myopic children in Singapore. Br J Ophthalmol 2005;89:1489-94.

27. Saw SM, Nieto FJ, Katz J, et al. Factors related to the progression of myopia in Singaporean children. Optom Vis Sci 2000;77:549-54.

28. Shen M, Fan F, Xue A, et al. Biomechanical properties of the cornea in high myopia. Vision Res 2008;48:2167-71.

29. Pruett RC. Progressive myopia and intraocular pressure: what is the linkage?: A literature review. Acta Ophthalmol 1988;66-117-27.

30. Medeiros FA, Meira-Freitas D, Lisboa R, et al. Corneal hysteresis as a risk factor for glaucoma progression: a prospective longitudinal study. Ophthalmology 2013;120:1533-40

31. Greene PR. Mechanical considerations in myopia: relative effects of accommodation, convergence, intraocular pressure, and the extraocular muscles. Am J Optom Physi Optic 1980;57:902-14.

32. McMonnies CW. Intraocular pressure spikes in keratectasia, axia myopia, and glaucoma. Optom Vis Sci 2008;85:1018-26.

33. Greene PR, McMahon TA. Scleral creep vs. temperature and pressure in vitro. Exp Eye Res 1979;29:527-37.

34. De Rousseau CJ, Bito LZ. Intraocular pressure of rhesus monkeys (Macaca mulatta). II.Juvenile ocular hypertension and its apparent relationship to ocular growth. Exp Eye Res 1981;32:407-17.

35. Read SA, Collins MJ, Annis-Brown T, et al. The short-term influence of elevated intraocular pressure on axial length. Ophthalmic Physiol Opt 2011;31:398-403.

36. Leydolt C, Findl O, Drexler W. Effects of change in intraocular pressure on axial eye length and lens position. Eye 2008;22:657-61.

37. McMonnies CW, Boneham GC. Corneal curvature stability with increased intraocular pressure. Eye \& Contact Lens 2007;33:130-7.

38. Gandhi PD, Gürses-Ozden R, Liebmann JM, et al. Attempted eyelid closure affects intraocular pressure measurement. Am J Ophthalmol 2001;131:417-20.

39. Bakke EF, Hisdal J, Semb SO. Intraocular pressure increases in parallel with systemic blood pressure during isometric exercise. Invest Ophthalmol Vis Sci 2009;50:760-4

40. Wang B, Naidu RK, Qu X. Factors related to axial length elongation and myopia progression in orthokeratology practice. PLoS One 2017:12:e0175913

41. Hiraoka T, Kakita T, Okamoto F. Long-Term effect of overnight orthokeratology on axial length elongation in childhood myopia: a 5-year follow-up studylong-term effect of orthokeratology on axial length. Invest Ophthalmol Vis Sci 2012;53:3913-9.

42. Charman WN, Mountford J, Atchison DA, et al. Peripheral refraction in orthokeratology patients. Optom Vis Sci 2006;83:641-8.

43. Moreno-Montan e's J, Maldonado MJ, Garci'a N, et al. Reproducibility and clinical relevance of the ocular response analyzer in nonoperated eyes: corneal biomechanical and tonometric implications. Invest Ophthalmol Vis Sci 2008;49:968-74

44. Hon Y, Cheung SW, Cho P, et al. Repeatability of corneal biomechanical measurements in children wearing spectacles and orthokeratology lenses. Ophthalmic Physiol Opt 2012;32:349-54.

45. Lim L, Gazzard G, Chan Y-H, et al. Cornea biomechanical characteristics and their correlates with refractive error in Singaporean children. Invest Ophthalmol Vis Sci 2008;49:3852-7.

46. Kamiya K, Hagishima M, Fujimura F, et al. Factors affecting corneal hysteresis in normal eyes. Graefes Arch Clin Exp Ophthalmol 2008;246:1491-4.

47. Kida T, Liu JH, Weinreb RN. Effects of aging on corneal biomechanical properties and their impact on 24-hour measurement of intraocular pressure. Am J Ophthalmol 2008;146:567-72.

48. Kirwan C, O'Keefe M, Lanigan B. Corneal hysteresis and intraocular pressure measurement in children using the reichert ocular response analyzer. Am J Ophthalmol 2006;142:990-2. 